\title{
Distributed Robust Vehicle State Estimation
}

\author{
Ehsan Hashemi, Mohamamd Pirani, Baris Fidan, Amir Khajepour, Shih-Ken Chen, Bakhtiar \\ Litkouhi
}

\begin{abstract}
A distributed estimation approach based on opinion dynamics is proposed to enhance the reliability of vehicle corners' velocity estimates, which are obtained by an unscented Kalman filter. The corners' estimates from a Kalman observer, which is formed by integrating the model-based and kinematic-based velocity estimation approaches, are utilized as opinions with different levels of confidence in the developed algorithm. More reliable estimates robust to disturbances and time delay are achieved via solving a convex optimization problem. Road tests confirm the robustness of the methods independent of the powertrain configuration on surfaces with various friction conditions in pure and combined-slip maneuvers, which are arduous for the current vehicle state estimators.
\end{abstract}

Keywords-Opinion dynamics, distributed estimation, unscented Kalman filter, robust observer design

\section{INTRODUCTION}

Developing a robust estimation structure to operate the available sensor data in production vehicles is a dominant objective of the car manufacturers. In this direction, reliable vehicle state estimation, which can benefit from cooperative and distributed estimation in intelligent transportation systems [1], at a reasonable cost, is essential for proper functioning of active safety systems in current vehicles.

The literature implemented two methods to estimated longitudinal and lateral velocity velocities: model-based and kinematic-based approaches. The model-based approach integrates measured longitudinal/lateral accelerations and uses tire forces to correct the estimation, but needs model parameters and surface friction. An EKF is employed for both longitudinal and lateral model-based vehicle state estimations in [2]-[4]; an EKF with smooth variable structure is also utilized in [5] to estimate lateral velocities and sideslip angles. Employing unscented Kalman filter (UKF), [6] and [7] propose different methods for estimation of the lateral and longitudinal velocities using knowledge of the road condition and tire model parameters, respectively. The approach in [8] applies a slidingmode observer for the velocity estimation and an EKF for estimation of the Burckhardt tire model's friction parameter. A model-based vehicle lateral state estimator is developed in [9] using a yaw rate gyroscope, a forward-looking monocular camera, an a priori map of road superelevation and temporally previewed lane geometry. On the other hand, the kinematic method uses acceleration and the yaw rate measurements from an inertial measurement unit (IMU) and estimates the vehicle velocities employing Kalman-based [10], [11], or nonlinear [12] observers. This method does not employ a tire model, but instead the sensors bias and noise should be identified precisely to have a reliable estimation. Two low-cost GPS receivers for the lateral velocity estimation is utilized in [13] and the the low update rate issue of conventional GPS receivers is compensated by combining the IMU and GPS data using an extended Kalman filter (EKF). [14] proposed a vehicle state estimator by combining data of magnetometer, GPS, and IMU and utilizing a stochastic filter integrated on the Kalman filter to reject disturbances in the magnetometer.

Reliable vehicle state estimation using conventional sensor measurement without using road friction information is desirable. Such a reliable estimator with corner-based structure is proposed in [15]. However, the developed observer is required to be augmented with a module to address reliability of the estimation setup. The proposed module is inspired by the opinion dynamics model [16], [17]. A four-node network is considered, whose members are four corners of the vehicle with estimated states as their opinions, each having a confidence level based on the corresponding corner's slip condition. A distributed estimation scheme is employed for the corners' states, estimated by a Kalman-based observer, to refine estimates and make them closer to that of the highconfidence corner.

The unscented Kalman filter estimator, which provides corners' estimates as input to the opinion dynamics, is first discussed in section II. The distributed estimation algorithm based on opinion dynamics is proposed in section III to provide velocities at corners and vehicle's CG using a reliability measure. Road experiments used to evaluate and verify the proposed approach on different road frictions and with various traction configurations are provided Section IV. Section V presents the conclusions.

\section{CORnERs' STATE Estimation By UnSCEnTED KALMAN FILTER}

The kinematic approach is combined with the tire's internal states at each corner in [15] to estimate longitudinal speed and to tackle uncertain model parameters and measurement noises. Because of the dynamics in its internal state, the lumped LuGre model [18], [19] has been selected and combined with the kinematics (accelerations at each corner) to estimate longitudinal velocity. The combined-slip LuGre model relates the longitudinal/lateral internal state $\bar{z}_{i}$ for $i \in\{x, y\}$ as $\dot{\bar{z}}_{i}=v_{r i}-C_{0 i} \bar{z}_{i}-\kappa_{i} R_{e}|\omega| \bar{z}_{i}$ where $C_{0 i}=\frac{\left\|M_{c}^{2} \mathbf{v}_{\mathbf{r}}\right\| \sigma_{0 i}}{g\left(\mathbf{v}_{\mathbf{r}}\right) \mu_{c i}^{2}}$, the rubber stiffness for longitudinal and lateral directions is $\sigma_{0 i}$, and $M_{c}=\left[\begin{array}{lll}\mu_{c x} & 0 ; 0 & \mu_{c y}\end{array}\right]$. The relative velocity vector $\mathbf{v}_{\mathbf{r}}=\left[\begin{array}{ll}v_{r x} & v_{r y}\end{array}\right]^{T}$ includes longitudinal and lateral relative velocities $v_{r x}=R_{e} \omega-v_{x t}$ and $v_{r y}=-v_{y t}$ where $v_{x t}$ and $v_{y t}$ are velocities in the tire coordinates. The vehicle stability is explored with the pure and combined-slip LuGre tire models in [20]. The force distribution along the patch line is represented by parameter $\kappa_{i}$. The transient region between the Coulomb and static friction in the combined-slip tire model is represented by $g\left(\mathbf{v}_{\mathbf{r}}\right)$. Replacing the road friction term $-C_{0 x} \bar{z}_{x}$ of the LuGre model with the bounded uncertainty $w_{1}$ related to the road friction condition, one can rewrite the LuGre longitudinal state dynamics as follows:

$$
\dot{\bar{z}}_{x}=v_{r x}-\kappa_{x} R_{e}|\omega| \bar{z}_{x}+w_{1} .
$$


The final form of the normalized longitudinal force $\mu_{x}$ of the averaged lumped LuGre model at each corner yields $\mu_{x}=$ $\sigma_{0} \bar{z}_{x}+\sigma_{1} \dot{\bar{z}}_{x}+\sigma_{2} v_{r x}$ where the rubber damping and relative viscous damping are denoted by $\sigma_{1 x}$ and $\sigma_{2 x}$, respectively. The derivatives of the relative velocities have also sensor noises and bias [21]:

$$
\dot{v}_{r x}=R_{e} \dot{\omega}-\dot{v}_{x t}+w_{2},
$$

in which the wheel's rotational acceleration is denoted by $\dot{\omega}$ and $\dot{v}_{x t}$ represents the projected longitudinal acceleration of the wheel's center in the tire coordinates. The term $w_{2}$ shows the deviation of the measured relative acceleration $R_{e} \dot{\omega}-\dot{v}_{x t}$ from $\dot{v}_{r x}$ because of measurement noises. Combining the modelbased states (1) and kinematic-based states (2), the proposed approach in [15] estimates relative velocities at each corner for $\omega>0$ by the following linear parameter-varying system with the longitudinal states $\mathbf{x}=\left[\begin{array}{lll}\bar{z}_{x} & v_{r x} & \dot{\bar{z}}_{x}\end{array}\right]^{T}$ :

$$
\begin{aligned}
& \dot{\mathbf{x}}=A_{c}(t) \mathbf{x}+B_{c} \mathbf{u}+\mathbf{w}_{p} \\
& \mathbf{y}=C_{c} \mathbf{x}+w_{m}
\end{aligned}
$$

in which $B_{c}=\left[\begin{array}{lll}0 & 1 & 1\end{array}\right]^{T}$, the estimation input is $\mathbf{u}=$ $R_{e} \dot{\omega}-\dot{v}_{x t}$, the output $\mathbf{y}=\mu_{x}$ is the normalized longitudinal force, which can be obtained from road friction-independent approaches using nonlinear and sliding mode observers [22][24], Kalman-based estimation [25], [26], and unknown input observers [27]-[29]. The measurement and process noises are denoted by $w_{m}$ and $\mathbf{w}_{p}=\left[\begin{array}{lll}w_{1} & w_{2} & \bar{w}_{1}\end{array}\right]^{T}$, respectively, with uncertainties $\bar{w}_{1}$ in the derivative of the longitudinal tire state. The output matrix is $C_{c}=\left[\begin{array}{lll}\sigma_{0 x} & \sigma_{2 x} & \sigma_{1 x}\end{array}\right]^{T}$, and $A_{c}(t)$ is defined by:

$$
A_{c}(t)=\left[\begin{array}{ccc}
-\kappa_{x} R_{e} \omega & 1 & 0 \\
0 & 0 & 0 \\
-\kappa_{x} R_{e} \dot{\omega} & 0 & -\kappa_{x} R_{e} \omega
\end{array}\right]
$$

The system (3) is a linear time-varying system with deterministic parameters, wheel speed and wheel acceleration. Uniform detectability and stabilizability conditions are investigated in the Appendix to check the stability of the proposed velocity estimators for known zero/nonzero initial states and complete uncertainty of the initial-state statistics.

Theorem 1: There exists a state estimator such as Kalman filter with bounded error covariance for the time-variant system (3) with deterministic time-varying parameters and known/ uncertain initial state and covariance.

Proof: The proof is provided in Section VI.

Consequently, the estimator (3) is observable and it is feasible to estimate the tire internal states $\bar{z}_{x}$ and the relative velocities $v_{r x}$ by a Kalman-based observer such as UKF [30], [31] with any known or stochastic initial covariance matrix at each corner $j$ where $j \in\{\mathrm{fL}, \mathrm{fR}, \mathrm{rL}, \mathrm{rR}\}$ shows the front-left, front-right, rear-left, and rear-right tires, respectively. The UKF employs a transformation, which introduces the Sigma vectors $\boldsymbol{\Sigma} \in \mathbb{R}^{\bar{N} \times 2 \bar{N}+1}$ ( $\bar{N}$ is the length of the state vectors) around $\mathbf{x}$, to include the nonlinear and non-Gaussian characteristics of the system that was a challenge for the longitudinal velocity estimation in [15]. Algorithm 1 shows the state estimation steps using the UKF. The square root factorization of the covariance matrix $\mathbf{P}_{k-1}$ is obtained by Cholesky decomposition at each time step $k$. Spread of the sigma points far from the mean

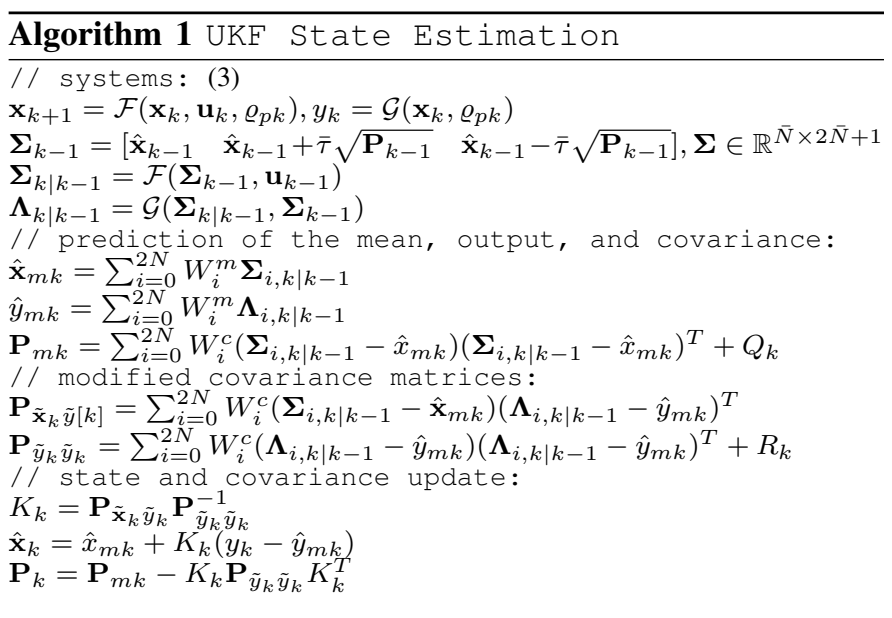

values of states are shown by the scalar $\bar{\tau}=\sqrt{\bar{N}+\bar{\eta}}$, where $\bar{\eta}$ is the compound scaling parameter $\bar{\eta}=\bar{\epsilon}^{2} \bar{N}-\bar{N}$ and $\bar{\epsilon}=\sqrt{3 / \bar{N}}$. The weighting parameters $W_{i}^{c}, W_{i}^{m}$ are defined by $W_{i}^{c}=W_{i}^{m}=\frac{1}{2}(\bar{N}+\bar{\eta})$ for all sets $i \in\{1,2, \ldots, 2 \bar{N}\}$ and $W_{0}^{c}=\frac{\bar{\eta}}{\bar{N}+\bar{\eta}}+1-\bar{\epsilon}^{2}+\bar{\beta}, W_{0}^{m}=\frac{\bar{\eta}}{\bar{N}+\bar{\eta}}$ for $i=0$ with the parameter $\bar{\beta}=2$. The estimated relative velocities $\hat{v}_{r x_{j}}$ at each corner $j$ from (3) are used for calculation of the corresponding values in the tire coordinates as $v_{x t_{j}}=R_{e} \omega_{j}-\hat{v}_{r x_{j}}$. The longitudinal speed at each corner in the vehicle's body coordinates is calculated by $v_{x_{j}}=v_{x t_{j}} \cos \delta-v_{y t_{j}} \sin \delta$ with $\delta$ as steering angle at front tires. Corners' opinion $\phi \in \mathbb{R}^{4}$ are defined by (5) using the mapped longitudinal velocities to $\mathrm{CG}$ and longitudinal velocity estimates $\mathbf{v}_{x}=\left[\begin{array}{llll}v_{x_{f L}} & v_{x_{f R}} & v_{x_{r L}} & v_{x_{r R}}\end{array}\right]^{T}$ at corners

$$
\phi=\mathbf{v}_{x}+\frac{r}{2} \mathbf{T}
$$

where the measured yaw rate is denoted by $r$ and the elements of $\phi$ are the mapped velocities from corner $j$ to the vehicle's CG, i.e., $\phi_{j}(t)$. Front and rear track widths $T_{f}, T_{r}$ are elements of $\mathbf{T}$ as in $\mathbf{T}=\left[\begin{array}{llll}-T_{f} & T_{f} & -T_{r} & T_{r}\end{array}\right]^{T}$.

Longitudinal velocity estimates are less reliable in highslip conditions due to less confidence in longitudinal force estimates $\mu_{x}$ (as the output) in (3). To address this issue, a robust distributed algorithm based on opinion dynamics is developed in this study which implements mapped corners' velocities to $\mathrm{CG}, \phi_{j}(t)$, and self-confidence of corners to their opinion, $\mathcal{W}_{j}>0$, based on the slip condition. Slip ratio is calculated at each corner using $\bar{\lambda}_{j}=\frac{R_{e} \omega_{j}-v_{x t_{j}}}{\max \left\{R_{e} \omega, v_{x t_{j}}\right\}}$ and is employed to show the level of confidence of the mapped corner velocities to the $\mathrm{CG}$ as (6) with constants $k_{1 x}, k_{2 x}>0$

$$
\mathcal{W}_{j}=k_{1 x}\left(k_{2 x}-\left|\bar{\lambda}_{j}\right|\right) \text {, }
$$

which confirms low reliance on corners with high-slip conditions. All corners' levels of confidence can be represented as a diagonal matrix $\mathcal{W}=\operatorname{diag}\left\{\mathcal{W}_{\mathrm{fL}}, \mathcal{W}_{\mathrm{fR}}, \mathcal{W}_{\mathrm{rL}}, \mathcal{W}_{\mathrm{rR}}\right\}$ for longitudinal estimates.

\section{DistribUted ESTIMATION BY OPINION DYNAMICS}

The selected Kalman-based velocity estimator at each corner and the confidence level based on the slip condition were introduced in the previous section. This section focuses 
on a distributed estimation approach robust to disturbances and delays to reach a reliable consensus among corner estimates. Corner estimated velocities are mapped to CG and denoted by the opinions $\phi_{j}(t)$ with confidence level $\mathcal{W}_{j}$ which is a monotonic decreasing function of the slip ratio $\bar{\lambda}_{j}$. The proposed dynamics for each opinion (in the longitudinal and lateral direction) is in the following form

$$
\dot{\phi}_{k}(t)=\sum_{j \in \mathcal{N}_{k}}\left(\phi_{k}(t)-\phi_{j}(t)\right)-\mathcal{W}_{j}\left(\phi_{k}(t)-\phi_{k}(0)\right),
$$

where $\mathcal{N}_{k}$ is the set of the neighbors of the $k$-th corner. Equation (7) in vector form changes to (8) with $\phi(t) \in \mathbb{R}^{4}$ for all states and $\mathbf{y}(t)$ as the output of interest.

$$
\begin{aligned}
& \dot{\boldsymbol{\phi}}(t)=-\bar{L} \boldsymbol{\phi}(t)+\mathcal{W} \boldsymbol{\phi}(0), \\
& \mathbf{y}(t)=\boldsymbol{\phi}(t),
\end{aligned}
$$

where $\bar{L}=L+\mathcal{W}$ in which $L$ is the (positive semi-definite) Laplacian matrix and $\mathcal{W}$ is a (positive definite) confidence level matrix. Consequently, $\bar{L}$ will be a positive definite matrix. The communication graph is a complete graph since each corner can communicate with other three ones. Thus, $\bar{L}$ yields

$$
\bar{L}=\left[\begin{array}{cccc}
3+\mathcal{W}_{\mathrm{fL}} & -1 & -1 & -1 \\
-1 & 3+\mathcal{W}_{\mathrm{fR}} & -1 & -1 \\
-1 & -1 & 3+\mathcal{W}_{\mathrm{rL}} & -1 \\
-1 & -1 & -1 & 3+\mathcal{W}_{\mathrm{rR}}
\end{array}\right]
$$

The complete graph with corners' longitudinal velocity estimates, structure of the developed distributed estimation scheme, and the hardware layout for real-time implementation

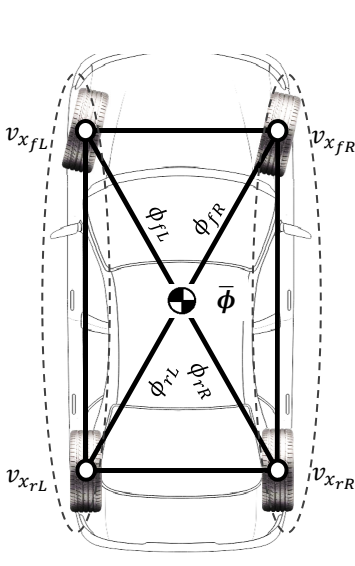

(a)

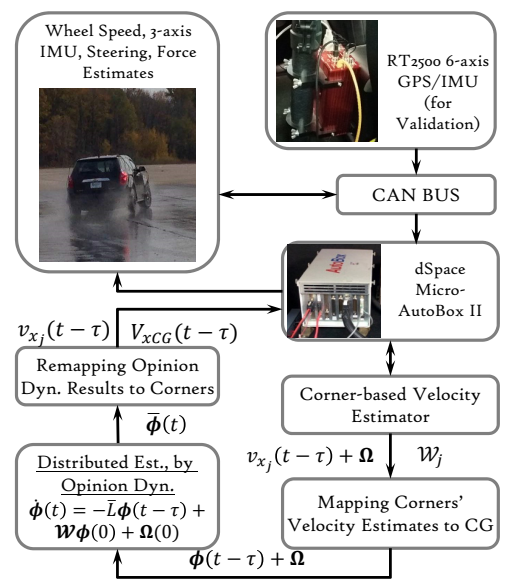

(b)
Fig. 1: (a) Complete graph (b) Distributed estimation by opinion dynamics and hardware layout

The following proposition shows how the steady-state value of opinion dynamics (8) is inside the convex hull of the longitudinal estimator's initial opinions.

Proposition 1: The states in opinion dynamics (8) asymptotically converge to a convex combination of the initial opinions.
Proof: The steady-state opinion for the longitudinal and lateral direction is

$$
\bar{\phi}=\bar{L}^{-1} \mathcal{W} \phi(0) \text {. }
$$

Having $L \mathbf{1}=\mathbf{0}$ results in $(\bar{L}-\mathcal{W}) \mathbf{1}=\mathbf{0}$ that yields $\bar{L} \mathbf{1}=$ $\mathcal{W} \mathbf{1}$. Left multiplying both sides by $\bar{L}^{-1}$, we get $\bar{L}^{-1} \mathcal{W} \mathbf{1}=$ 1. This shows that $\bar{L}^{-1} \mathcal{W}$ is a row stochastic matrix and (10) converges to some convex combinations of the elements of $\phi(0)$.

Remark 1: Based on Proposition 1, the steady-state modes of the corners' opinion converge to some points inside the convex hull of the initial opinion; hence, the variation of corners' opinion decreases during the opinion dynamics. Moreover, final opinion values become closer to the ones with higher confidence level.

Due to the existence of disturbances $\Omega(t)$ and time delay $\tau$ (caused by low-pass filters applied to signals $\dot{\omega}, \dot{v}_{x t}$ and measured accelerations), (8) can be written as

$$
\dot{\phi}(t)=-\bar{L} \phi(t-\tau)+\mathcal{W} \phi(0)+\Omega(t) .
$$

The following lemmas discuss the stability of dynamics (8) under time delay and its $\mathcal{H}_{\infty}$ robustness to external disturbances.

Lemma 1 ( [32]): The system $\mathcal{H}_{\infty}$ norm of the dynamics (8) is $\frac{1}{\lambda_{1}(\bar{L})}$.

Lemma 2 ( [33]): The opinion dynamics (11) is asymptotically stable in the presence of constant time delay $\tau \in$ $\left[0, \tau_{\max }\right]$ if and only if

$$
\tau_{\max }<\frac{\pi}{2 \lambda_{4}(\bar{L})}
$$

Lemmas 1 and 2 reveal that the robustness of (8) depends on the smallest and largest eigenvalues of $\bar{L}$. According to Lemmas 1 and 2, there exists a trade-off in choosing high gains (confidence values) to meet both robustness metrics to disturbances and time delay. Increasing $\lambda_{1}(\bar{L})$ (increasing robustness to disturbance) results in increasing $\lambda_{4}(\bar{L})$ (decreasing robustness to time delay), and vice versa. In order to tackle this problem, confidence matrices $\mathcal{W}$ should be chosen such that both robustness requirements are satisfied to a certain level. More formally, the following optimization problem is introduced:

$$
\begin{array}{cl}
\underset{\mathcal{W}}{\operatorname{minimize}} & J(\mathcal{W})=-\lambda_{1}(\bar{L}) \\
\text { subject to } & \lambda_{4}(\bar{L}) \leq \gamma \\
& \mathcal{W}_{j} \in\left[\mathcal{W}_{j_{\min }}, \mathcal{W}_{j_{\max }}\right]
\end{array}
$$

for some $\gamma$, where $\mathcal{W}_{j_{\min }}, \mathcal{W}_{j_{\max }}>0$ are determined based on the slip condition of the corresponding corner and (6). Here $\gamma$ is a design parameter which represents the inherent existing delay in the state estimator setup due to filtering. We know that the spectral radius of a positive definite matrix is a convex function and its smallest eigenvalue is a concave function of the matrix elements. Hence, the above problem is a convex problem and has a unique optimal solution. In practice and road experiments, signals are used in discrete-time. The discretization of the continuous-time systems discussed here is done by the step-invariance method [34], because of its precision and response characteristics. In this direction, the 


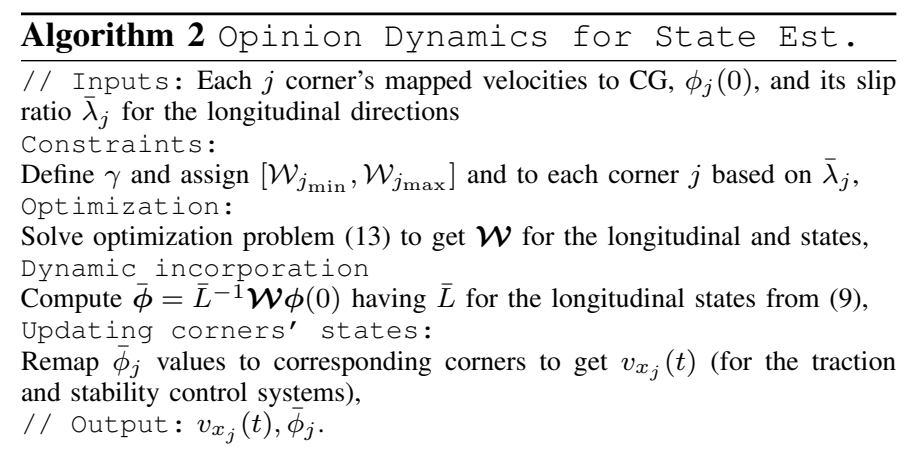

velocity estimators and the opinion dynamics are implemented in the discrete-time.

Algorithm 2 and Figure 1-b provide the detail and general structure of the utilized opinion dynamics. To validate the longitudinal velocity estimator with the proposed opinion dynamics, an RT2500 6-axis GPS system is used. The measured accelerations, yaw rate, wheel speed, and steering angle are inputs to the corner-based velocity estimator. Real-time acquisition and processing of sensory information and the developed algorithm is done using the dSPACE $\mathrm{R}$ MicroAutobox with CAN-bus communication. The dSPACE compiles measurements for estimators and controllers in MATLAB/Simulink. The sampling frequency the experiment is set to be $200[\mathrm{~Hz}]$.

\section{RESUlTS AND DisCUSSION}

To validate the proposed distributed state estimation algorithm on an electrified instrumented SUV, road experiments are conducted on surfaces with various friction conditions and in different maneuvers. Experimental results are provided in this section. The vehicle specifications are: wheel's moment of inertia $I_{w}=1.7 \mathrm{kgm}^{2}$, mass $m=2045 \mathrm{~kg}$, moment of inertia $I_{z}=4161 \mathrm{kgm}^{2}$, and front/rear track widths $T_{f}=$ $1.59, T_{r}=1.58 \mathrm{~m}$. Constants for the confidence level in (6) for the road experiments are $k_{1 x}=25$ and $k_{2 x}=1$. For the velocity estimator, the load distribution factor is $\kappa_{x}=7.6$, the rubber stiffness and damping are $\sigma_{0 x}=6581 / \mathrm{m}$ and $\sigma_{1 x}=0.75 \mathrm{~s} / \mathrm{m}$, respectively, and the relative viscous damping is $\sigma_{2 x}=0.0018$. The distributed opinion dynamics scheme on the Kalman-based longitudinal state estimator is examined in a severe launch on an icy surface and the results are illustrated in Fig. 2 for the AWD powertrain configuration. The wheel peripheral translational velocity, "Wheel Periph.", increases drastically for all four wheels due to high wheel speed on the icy surface, but the estimated wheel center velocity is accurate when compared to the measured one by GPS.

Coping with model uncertainties and time delay, the unscented Kalman filter and the optimized corners' final confidence levels lead to the observed smooth and accurate velocity estimation at corners for such highly slippery surface with $\mu \approx 0.25$. To check the performance of the augmented Kalman-based state estimator by the robust distributed estimation scheme in a laterally-excited maneuver, a lane-change scenario with RWD powertrain configuration is conducted on a surface covered with packed snow, $\mu \approx 0.4$, and the longitudinal velocity estimation results are provided in Fig. 3.
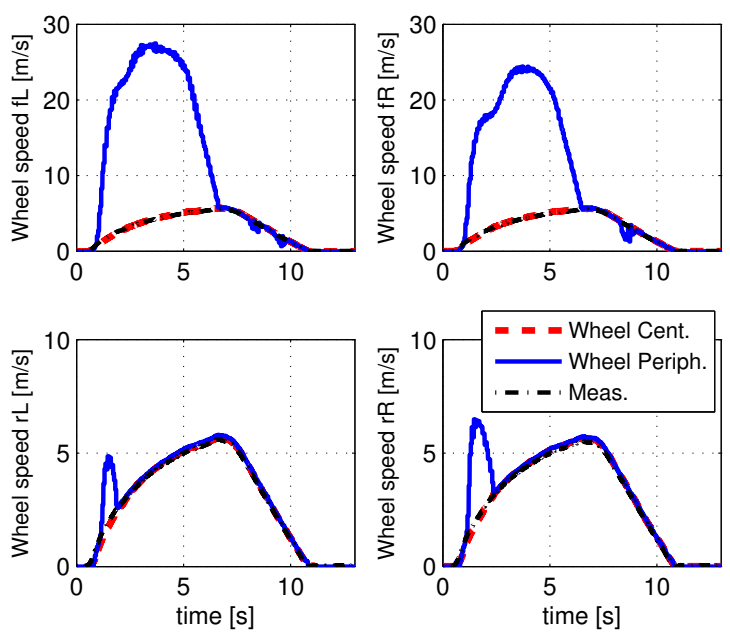

Fig. 2: Estimated velocities for AWD, accel./brake on ice.
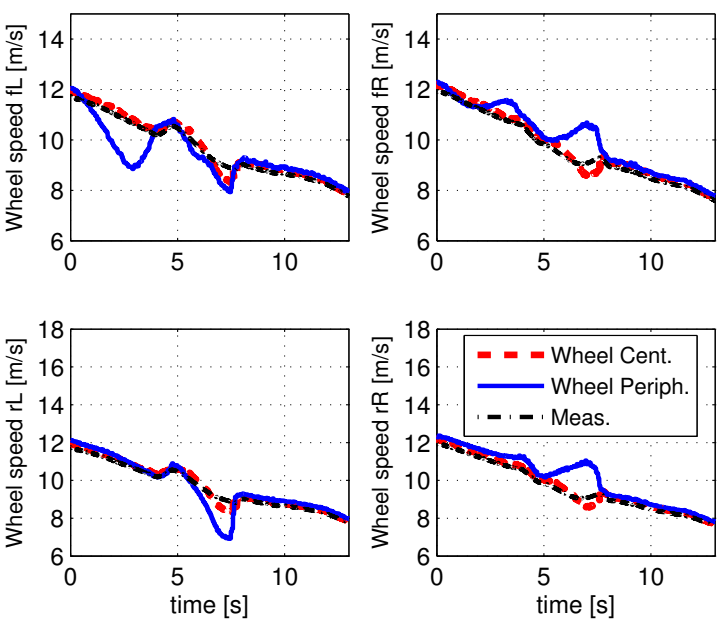

Fig. 3: Estimated velocities for RWD, LC on snow.

Several rises and drops in the "Wheel Periph." velocity compared to the measured wheel center velocity, "Meas.", are observed at each corner due to high-slip cases, but the developed state estimator, "Wheel Cent.", with the incorporated robust opinion dynamics exhibit good performance even for such laterally-excited maneuver on snow with RWD traction configuration.

Combined-slip maneuvers are demanding for model-based vehicle state estimators because of several model's contributing parameters and the road friction coefficient, which are unknown in practice. A combined-slip acceleration-in-turn (AiT), in which the capacity of a tire in longitudinal/lateral directions reduces due to high slip in another direction, is done for the test vehicle with RWD configuration and results are illustrated in Figure 4. The test is performed on wet sealed asphalt with $\mu \approx 0.45$. The measured longitudinal/lateral accelerations together with the wheel speed at each corner are provided in Fig. 4 to show the high-slip and arduous characteristics of the maneuver. 

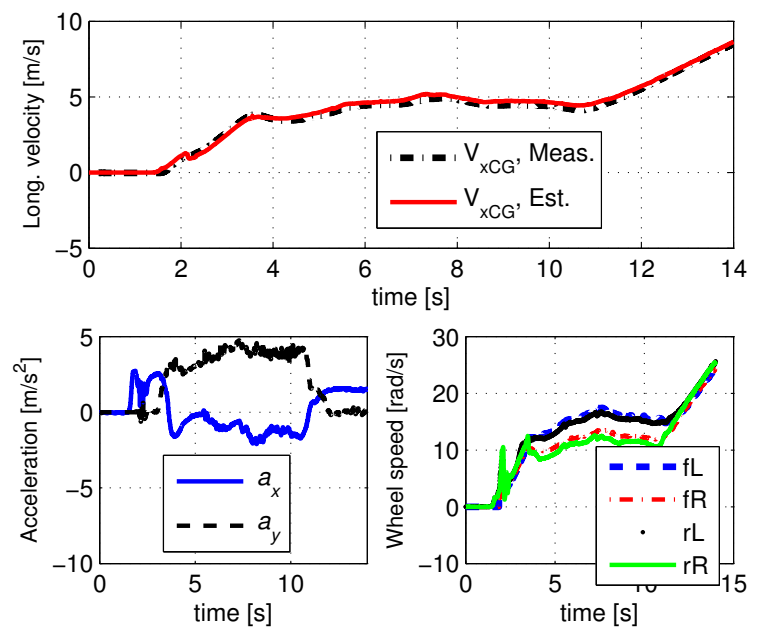

Fig. 4: Estimated velocities for RWD, AiT on wet sealer.

Figure 4 demonstrates good performance of distributed state estimator (based on the opinion dynamics) to handle combined-slip conditions despite several high-slip cases near $t=2$ and $t=3.4 \mathrm{~s}$ for right tires due to the load transfer to the left side and reduced tire capacities.

To check the performance of the distributed state estimator in combined-slip conditions with another traction configuration and on friction-varying surfaces, another acceleration-in-turn with transitions from wet sealer to dry asphalt is done and the estimation outcomes are shown in Fig. 5. The implemented robust opinion dynamics on the outcomes of the Kalman-based longitudinal velocity estimator shows consistent results with FWD traction configuration even with changes in the road friction condition.
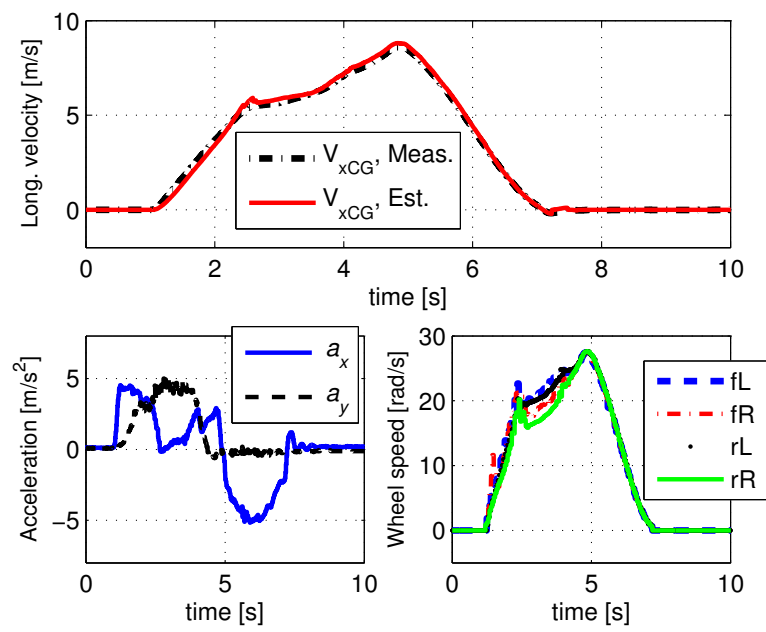

Fig. 5: Estimated velocities for FWD, AiT on wet sealer.

Figure 5 demonstrates good performance of the estimator validated by the measurement from an accurate GPS at the vehicle's CG. In spite of rises in corners' wheel speed due to the slippery surface and reduction in tire capacities, the developed algorithm shows reliable longitudinal speed estimates.

\section{CONClusion}

A distributed estimation algorithm based on opinion dynamics is presented in this article to increase the reliability of a Kalman-based vehicle velocity estimator. Utilizing an unscented Kalman filter on an integrated model-based and kinematic-based state estimation scheme, the proposed velocity observer provides velocity at corners and the level of confidence characterised by the magnitude of slip ratio of that particular corner. The mapped corners' velocity to CG was interpreted as the opinion of that corner in a linear timeinvariant dynamical system to attenuate the effect of disturbances and time delay in real-time implementation. Improved estimated states, which are closer to the network element(s) with the highest confidence level, are remapped to each corner to make the overall estimation setup performing more reliably. To validate the approach, several road experiments have been conducted on surface with various friction conditions and in normal and harsh driving scenarios.

\section{APPENDIX}

Proof of Theorem 1: The time-variant longitudinal state estimator (3) is discretized by the step-invariance method in which the discrete-time system has the output matrix $C_{d}=C_{c}$ and state/input matrices $A=e^{A_{c}(t) T_{s}}, B=$ $\int_{0}^{T_{s}} e^{A_{c}(t) \tau} B_{c}(t) d \tau$. The discrete-time form yields:

$$
\begin{aligned}
\mathbf{x}_{k+1} & =A_{k} \mathbf{x}_{k}+B_{k} \mathbf{u}_{k}+\varrho_{p k} \\
\mathbf{y}_{k} & =C_{k} \mathbf{x}_{k}+\varrho_{m k},
\end{aligned}
$$

with process and measurement uncertainties $\varrho_{p k}, \varrho_{m k}$, which have the covariances $Q_{k}=\mathbb{E}\left[\varrho_{p k}, \varrho_{p k}{ }^{T}\right], R_{k}=\mathbb{E}\left[\varrho_{m k}, \varrho_{m k}{ }^{T}\right]$, accordingly. Process and measurement noises are assumed to be uncorrelated $\mathbb{E}\left[\varrho_{p k}, \varrho_{m k}^{T}\right]=0$. Uniform detectability leads to bounded error covariance. The stochastic observability, stability and convergence of the state mean, and bounds on error covariance of the Kalman estimator on LTV systems, such as that in the discretized form of (3), were studied in [35]-[37].

Lemma 3: [35], [37] With the known initial state/covariance and uniform detectability of $\left[A_{k}, C_{k}\right]$ in (A1) with process and measurement noise covariances $Q_{k}, R_{k}$, there exists a state estimator such as the Kalman having bounded error covariance. Furthermore, stabilizability of the pair $\left[A_{k}, \bar{G}_{k}\right]$ leads to exponential stability of the Kalman estimator, where $\bar{G}_{k}$ is an appropriate matrix obtained by $Q_{k}=\bar{G}_{k} \bar{G}_{k}^{T}$.

According to Lemma 3, the detectability condition is checked and satisfied for the discrete-time form of the proposed estimators (3). The bounded error covariance and stability of the Kalman estimator for systems with completely uncertain initial covariance/states is investigated in [38]. The following Lemma addresses the stochastic initial state condition.

Lemma 4: [38] The Kalman estimator on the system (A1) with an error covariance matrix and stochastic initial state $\mathbf{P}_{0 \mid-1}=\psi I, \psi \in \mathbb{R}^{+}$is stochastically observable if the 
conditions (A2) holds for a finite time $t_{f}$ and for $t_{k} \geq t_{f}$ with the predefined bound $\bar{\sigma}_{b}$ :

$$
\bar{\sigma}_{\max }\left(\mathcal{M}_{f}\right)=0, \quad \bar{\sigma}_{\max }\left(\mathcal{N}_{k+1}\right)<\bar{\sigma}_{b}
$$

where $\mathcal{M}, \mathcal{N}$ are obtained from a modified Riccati equation as provided in [15].

The two criteria (A2) are examined using $Q=3^{-3} I_{3 \times 3}$ and $\bar{R}_{x}=0.0015$ for the longitudinal velocity estimators and met with the model parameters provided in section IV in various driving scenarios and road conditions. For all performed road experiments $\bar{\sigma}_{\max }\left(\mathcal{M}_{f}\right)$ converges to zero after at most $0.029 \mathrm{~s}$ and $\bar{\sigma}_{b}=3.4$. Therefore, the Kalman observer is stable even if the scalar $\psi$ has infinite values.

\section{ACKNOWLEDGMENT}

This work was supported by Automotive Partnership Canada, Ontario Research Fund, and the General Motors Co. [grant numbers APCPJ 395996-09 and ORF-RE-04-039].

\section{REFERENCES}

[1] N. Lu, N. Cheng, N. Zhang, X. Shen, and J. W. Mark, "Connected vehicles: Solutions and challenges," IEEE Internet of things journal, vol. 1, no. 4, pp. 289-299, 2014.

[2] M. Satria and M. Best, "Comparison between Kalman Filter and Robust Filter for Vehicle Handling Dynamics State Estimation," in $S A E$ Technical Paper 2002-01-1185, 2002.

[3] T. a. Wenzel, K. J. Burnham, M. V. Blundell, and R. a. Williams, "Dual Extended Kalman Filter for Vehicle State and Parameter Estimation," Vehicle System Dynamics, vol. 44, no. 2, pp. 153-171, 2006.

[4] L. Tong, "An Approach for Vehicle State Estimation Using Extended Kalman Filter," in Syst. Simul. Sci. Comput., vol. 1, 2012, pp. 56-63.

[5] X. Huang and J. Wang, "Robust Sideslip Angle Estimation for Lightweight Vehicles Using Smooth Variable Structure Filter," in ASME Dynamic Systems and Control Conference, 2013, pp. 1-8.

[6] M. Wielitzka, M. Dagen, and T. Ortmaier, "State estimation of vehicle's lateral dynamics using unscented kalman filter," in Decision and Control (CDC), 2014 IEEE 53rd Annual Conference on. IEEE, 2014, pp. 50155020.

[7] F. Sun, K. Lolenko, and J. Rudolph, "Nonlinear observer design for state estimation during antilock braking," Proceedings of the Institution of Mechanical Engineers, Part I: Journal of Systems and Control Engineering, vol. 228, no. 2, pp. 78-86, 2014.

[8] X. Zhang, Y. Xu, M. Pan, and F. Ren, "A vehicle ABS adaptive slidingmode control algorithm based on the vehicle velocity estimation and tyre/road friction coefficient estimations," Vehicle System Dynamics, vol. 52, no. 4, pp. 475-503, 2014.

[9] A. A. Brown and S. N. Brennan, "Lateral vehicle state and environment estimation using temporally previewed mapped lane features," IEEE Transactions on Intelligent Transportation Systems, vol. 16, no. 3, pp. 1601-1608, 2015.

[10] J. Ryu, "State and Parameter Estimation for Vehicle Dynamics Control using GPS," Ph.D. dissertation, Stanford University, 2004.

[11] J. Ryu and J. C. Gerdes, "Integrating inertial sensors with global positioning system (gps) for vehicle dynamics control," Journal of Dynamic Systems, Measurement, and Control, vol. 126, no. 2, pp. 243254, 2004.

[12] L. Imsland, T. A. Johansen, T. I. Fossen, H. F. Grip, J. C. Kalkkuhl, and A. Suissa, "Vehicle velocity estimation using nonlinear observers," Automatica, vol. 42, no. 12, pp. 2091-2103, 2006.

[13] J.-H. Yoon and H. Peng, "A cost-effective sideslip estimation method using velocity measurements from two gps receivers," Vehicular Technology, IEEE Transactions on, vol. 63, no. 6, pp. 2589-2599, 2014.

[14] _ - "Robust vehicle sideslip angle estimation through a disturbance rejection filter that integrates a magnetometer with gps," IEEE Transactions on Intelligent Transportation Systems, vol. 15, no. 1, pp. 191-204, 2014.
[15] E. Hashemi, A. Kasaiezadeh, S. Khosravani, A. Khajepour, N. Moshchuk, and S.-K. Chen, "Estimation of longitudinal speed robust to road conditions for ground vehicles," Vehicle System Dynamics, vol. 54, no. 8, pp. 1120-1146, 2016.

[16] A. Clark, B. Alomair, L. Bushnell, and R. Poovendran, Submodularity in Dynamics and Control of Networked Systems. Springer, 2016.

[17] M. Mesbahi and M. Egerstedt, Graph Theoretic Methods in Multiagent Networks. Princeton University Press, 2010.

[18] C. Canudas-de Wit, P. Tsiotras, E. Velenis, M. B. Gissinger, and G. Gissinger, "Dynamic Friction Models for Road/Tire Longitudinal Interaction," Vehicle System Dynamics, vol. 39, pp. 189-226, 2003.

[19] E. Velenis, P. Tsiotras, C. Canudas-de Wit, and M. Sorine, "Dynamic tyre friction models for combined longitudinal and lateral vehicle motion," Vehicle System Dynamics, vol. 43, no. 1, pp. 3-29, 2005.

[20] E. Hashemi, M. Pirani, A. Khajepour, and A. Kasaiezadeh, "A comprehensive study on the stability analysis of vehicle dynamics with pure/combined-slip tyre models," Vehicle system dynamics, vol. 54, no. 12, pp. 1736-1761, 2016.

[21] Y. Wang and D. Bevly, "Longitudinal Vehicle State Estimation; General Motors Summary Report," General Motors Summary Report, Tech. Rep., 2010.

[22] N. Patel, C. Edwards, and S. Spurgeon, "Tyreroad friction estimationa comparative study," Proceedings of the Institution of Mechanical Engineers, Part D: Journal of Automobile Engineering, vol. 222, no. 12, pp. 2337-2351, 2008.

[23] G. Baffet, A. Charara, and D. Lechner, "Estimation of vehicle sideslip, tire force and wheel cornering stiffness," Control Engineering Practice, vol. 17, no. 11, pp. 1255-1264, 2009 .

[24] R. Rajamani, G. Phanomchoeng, D. Piyabongkarn, and J. Y. Lew, "Algorithms for real-time estimation of individual wheel tire-road friction coefficients," Mechatronics, IEEE/ASME Transactions on, vol. 17, no. 6, pp. 1183-1195, 2012.

[25] M. Doumiati, A. Victorino, D. Lechner, G. Baffet, and A. Charara, "Observers for vehicle tyre/road forces estimation: experimental validation," Vehicle System Dynamics, vol. 48, no. 11, pp. 1345-1378, 2010.

[26] E. Hashemi, A. Kasaeizadeh, A. Khajepour, N. Mushchuk, and S.-K. Chen, "Robust Estimation and Experimental Evaluation of Longitudinal Friction Forces in Ground Vehicles," in ASME IMECE2014, 2014.

[27] S. Mammar, S. Glaser, and M. Netto, "Vehicle lateral dynamics estimation using unknown input proportional-integral observers," in American Control Conference, 2006. IEEE, 2006, pp. 6-pp.

[28] Y. Wang, D. M. Bevly, and S.-k. Chen, "Lateral tire force estimation with unknown input observer," in ASME 2012 5th Annual Dynamic Systems and Control Conference joint with the JSME 2012 11th Motion and Vibration Conference. American Society of Mechanical Engineers, 2012, pp. 531-538.

[29] E. Hashemi, M. Pirani, A. Khajepour, B. Fidan, A. Kasaiezadeh, S. Chen, and B. Litkouhi, "Integrated estimation structure for the tire friction forces in ground vehicles," in Advanced Intelligent Mechatronics (AIM), 2016 IEEE International Conference on. IEEE, 2016, pp. $1657-1662$.

[30] S. Julier and J. Uhlmann, "A New Extension of the Kalman Filter to Nonlinear Systems," in The 11th International Symposium on Aerospace/Defense Sensing, Simulation and Controls, 1997.

[31] R. V. D. Merwe and E. Wan, "Efficient Derivative-Free Kalman Filters for Online Learning." in European Symposium on Artificial Neural Networks, ESANN, 2001, pp. 205-210.

[32] M. Pirani, E. M. Shahrivar, and S. Sundaram, "Coherence and convergence rate in networked dynamical systems," Proceedings of $C D C$ 2015, the 54th IEEE Conference on Decision and Control, 2015.

[33] M. Buslowicz, "Simple stability criterion for a class of delay differential systems," International Journal of Systems Science, vol. 18, pp. 993995, 1987.

[34] G. F. Franklin, J. D. Powell, and M. L. Workman, Digital Control of Dynamic Systems. Menlo Park: Addison Wesley Longman, 1998.

[35] B. Anderson and J. B. Moore, "Detectability and stabilizability of timevarying discrete-time linear systems," SIAM Journal on Control and Optimization, vol. 19, no. 1, pp. 20-32, 1981. 
[36] B. Delyon, "A note on uniform observability," Automatic Control, IEEE Transactions on, vol. 46, no. 8, pp. 1326-1327, 2001.

[37] R. F. Stengel, Optimal control and estimation. Courier Corporation, 2012.

[38] V. L. Bageshwar, D. Gebre-Egziabher, W. L. Garrard, and T. T. Georgiou, "Stochastic observability test for discrete-time kalman filters," Journal of Guidance, Control, and Dynamics, vol. 32, no. 4, pp. 13561370, 2009. 